\title{
Commercial Bovine Proteoglycan Is Highly Arthritogenic and Can Be Used as an Alternative Antigen Source for PGIA Model
}

\author{
Larissa Lumi Watanabe Ishikawa, Priscila Maria Colavite, \\ Larissa Camargo da Rosa, Bianca Balbino, \\ Thais Graziela Donegá França, Sofia Fernanda Gonçalves Zorzella-Pezavento, \\ Fernanda Chiuso-Minicucci, and Alexandrina Sartori
}

Department of Microbiology and Immunology, Biosciences Institute, São Paulo State University (UNESP), 18618-000 Botucatu, SP, Brazil

Correspondence should be addressed to Larissa Lumi Watanabe Ishikawa; larissalumi@gmail.com

Received 28 February 2014; Revised 29 April 2014; Accepted 1 May 2014; Published 27 May 2014

Academic Editor: Yehuda Shoenfeld

\begin{abstract}
Copyright ( 92014 Larissa Lumi Watanabe Ishikawa et al. This is an open access article distributed under the Creative Commons Attribution License, which permits unrestricted use, distribution, and reproduction in any medium, provided the original work is properly cited.
\end{abstract}

\begin{abstract}
Rheumatoid arthritis (RA) is the most common systemic autoimmune disease. It affects mainly the joints, causing synovitis, cartilage destruction, and bone erosion. Many experimental models are used to study the mechanisms involved in immunopathogenesis and new therapies for this disease. Proteoglycan-induced arthritis (PGIA) is a widely used model based on the cross-reactivity of injected foreign (usually human) PG and mice self-PG. Considering the complexity of the extraction and purification of human PG, in this study we evaluated the arthritogenicity of bovine PG that is commercially available. Bovine PG was highly arthritogenic, triggering $100 \%$ incidence of arthritis in female BALB/c retired breeder mice. Animals immunized with bovine PG presented clinical symptoms and histopathological features similar to human RA and other experimental models. Moreover, bovine PG immunization determined higher levels of proinflammatory and anti-inflammatory cytokines in arthritic mice compared to healthy ones. As expected, only the arthritic group produced IgG1 and IgG2a antibodies against PG. Thus, commercial bovine PG can be used as an alternative antigenic source to PGIA for the study of many RA aspects, including the immunopathogenesis of the disease and also the development of new therapies.
\end{abstract}

\section{Introduction}

Rheumatoid arthritis (RA) is a chronic inflammatory disease that affects around 0.3 to $1 \%$ of the world population, with lower prevalence in developing countries [1]. It is considered the most common systemic autoimmune disease that usually affects the small joints, especially fingers. It may also involve larger joints, including shoulders, elbows, knees, and ankles. The inflammatory process in the joint is characterized by synovitis, cartilage destruction, and bone erosion. There is still no consensus on the autoantigens involved in this disease. Currently, it is known that some autoantigens such as cartilage components, chaperone proteins, enzymes, nuclear proteins, and citrullinated proteins might be involved $[2,3]$. Among several cell types found in the inflamed joint, CD4+ T-cells' subsets are considered the most important cells involved in synovitis and RA development [4]. Activated macrophages are also a very relevant source of inflammatory mediators, including proinflammatory cytokines [5]. TNF$\alpha$ and IL-1, for example, promote the accumulation of inflammatory cells in the joints and the synthesis of other cytokines, chemokines, and matrix metalloproteinases [6]. Many cytokines, including IL- 8 , TNF- $\alpha$, and IFN- $\gamma$, have been detected in synovial fluid. These cytokines, especially 
TNF- $\alpha$, activate resident synovial cells that by producing proteolytic enzymes mediate the destruction of joint cartilage, ligaments, and tendons. Recently, the presence of IL-17 at the site of inflammation and its synergistic effect with TNF- $\alpha$, exacerbating the inflammatory process, have been evidenced [7]. The participation of B cells in RA has been more investigated in recent years. The production of autoantibodies and cytokines, presentation of autoantigens to T cells, and ectopic lymphoid organogenesis are their possible roles [8]. Regulatory $\mathrm{T}$ cells have also been widely studied in both human and experimental arthritis because of their therapeutic and prophylactic potential [9].

There are several experimental models of arthritis being used to elucidate the mechanisms involved in the immunopathogenesis of RA. Also, the animal models are essential to study new therapy targets for this disease. Historically, the first experimental models of arthritis, which are called adjuvant-induced arthritis (AIA), were based on the inoculation of mycobacterial components suspended in mineral oil. Later, it was discovered that this model could be improved by using pristane, which is a purified component of mineral oil. The disease caused by pristane was more similar to human RA and this model has been widely used [10-12]. After that, there was an increased interest in experimental models based on the inoculation of cartilage components such as type II collagen and proteoglycan. These models presented clinical, immunological, histopathological, and genetic characteristics typical from human RA and, for that reason, they were considered the best models to study mechanisms and possible treatments for arthritis [13, 14]. In the 90s, the first arthritis transgenic models were described. By using immunogenetic tools, Keffer et al. [15] observed a spontaneous arthritis in mice overexpressing human TNF- $\alpha$ transgene. In this study, the animals developed a chronic inflammatory polyarthritis that evidenced the critical role of TNF- $\alpha$ in the immunopathogenesis of RA. Currently, collagen-induced arthritis (CIA) is a very reliable and reproducible experimental model that is being widely used for the study of all aspects of arthritis, including the immunopathogenesis of RA, the development of new drugs from natural extracts, the new molecular targets for treatment, and also gene therapy [16-19].

The experimental model chosen for this study was based on the immunization of $\mathrm{BALB} / \mathrm{c}$ mice with proteoglycan (PG). Proteoglycan-induced arthritis (PGIA) was elegantly described by Glant et al. [13]. Briefly, the systemic autoimmune arthritis in this model is induced by intraperitoneal inoculation of $\mathrm{BALB} / \mathrm{c}$ or $\mathrm{C} 3 \mathrm{H}$ mice with $\mathrm{PG}$ isolated from various sources. Many genetic and immunological aspects of PGIA have already been studied in this model. For example, epitopes recognized by the arthritogenic $\mathrm{T}$ cells and the contribution of various cytokines such as IFN- $\gamma$, IL- 4 , and IL-12 were determined [20-22]. Although human cartilage is the preferable source of $\mathrm{PG}$, its extraction and purification is a complex and laborious process that includes a variety of biochemical steps. Besides, ethical issues and rules involving the utilization of biological samples from human and animals contribute to complicate PG purification. In this scenario, we investigated the possible arthritogenicity of bovine PG in BALB/C mice. We considered that this evaluation could be very beneficial to researchers that are not able to purify human PG. Commercial availability of bovine PG could not only facilitate the experimental model implementation but also facilitate the comparison of results obtained by different laboratories.

\section{Materials and Methods}

2.1. Animals. Female BALB/c retired breeder (beyond the reproductive age) mice were removed from breeding colonies by the age of $8-11$ months and purchased from CEMIB (Campinas, SP, Brazil). They were maintained in the Department of Microbiology and Immunology facility under controlled conditions of luminosity ( $12 \mathrm{~h}$ light $/ 12 \mathrm{~h}$ dark) and temperature $\left(22 \pm 2^{\circ} \mathrm{C}\right)$. Mice were allocated in ventilated cages with sterile pine shavings and received sterile food and filtrated water ad libitum. The manipulation of the animals was in compliance with the local ethics committee (Protocol number 257-CEEA).

2.2. Arthritis Induction and Score Evaluation. As previously described [23], with minor modifications, a dose $(100 \mu \mathrm{L})$ containing $100 \mu \mathrm{g}$ of bovine proteoglycan extracted from nasal septum (Sigma Aldrich, St. Louis, MO, USA) and $1 \mathrm{mg}$ of emulsified (micelle form) dodecyl dioctadecyl ammonium bromide (DDA) adjuvant (Sigma Aldrich, St. Louis, MO, USA) was intraperitoneally injected three times with 21day interval for arthritis induction. After the third injection, arthritis score was daily evaluated until euthanasia (70 days after the first immunization). Arthritis severity was determined using a standard visual scoring system based on the degree of swelling and redness ranging from 0 to 4 for each paw. The following system was used: $0=$ normal; $1=$ mild swelling in the paw or one joint; 2 = moderate swelling and redness in the paw and one or more joints; 3 = pronounced swelling and redness in the paw, all joints, and ankle; $4=$ severe swelling and redness of the entire paw and ankle and movement limitation. This classification resulted in a total score that ranged from 0 to 16 for each animal.

2.3. Histopathological Analysis. After euthanasia, mice paws were collected and fixed in $10 \%$ formalin phosphate buffer for at least seven days at room temperature. The samples were thoroughly demineralized in 10\% Titriplex EDTA disodium salt (Merck Millipore, Darmstadt, Germany) for one to two months. The decalcified tissues were trimmed, dehydrated in graded ethanol, and embedded in paraffin. Serial sections $(5 \mu \mathrm{m})$ were cut and mounted on glass slides precoated with $0.1 \%$ poly-L-lysine (Sigma Aldrich, St. Louis, MO, USA). Histological assessment was carried out following routine hematoxylin and eosin (HE) staining. The images were acquired by a digital camera attached to the optical microscope (Nikon, Kurobanemuko, Otawara, Japan).

2.4. Immune Responses Evaluation. For cellular immune response, spleens were collected after euthanasia and the cells resuspended in RPMI medium containing gentamicin 
and fetal calf serum. The cells were stimulated with ConA $(5 \mu \mathrm{g} / \mathrm{mL})$ and PG $(50 \mu \mathrm{g} / \mathrm{mL})$. After 48 hours incubation at $37^{\circ} \mathrm{C} / 5 \% \mathrm{CO}_{2}$, the supernatants were collected for detection of IL-2, IL-6, TNF- $\alpha$, IL-17, IFN- $\gamma$, IL-5, and IL-10. These cytokines were quantified using enzyme linked immunosorbent assay (ELISA), according to the manufacturer's instructions (BD Biosciences, San Jose, CA, USA, and RD Systems, Minneapolis, MN, USA). For humoral immune response, blood samples were collected by facial vein two days before each dose and seven days after the third dose of PG+DDA. 70 days after the first immunization, the blood was collected by cardiac puncture. The sera were obtained by blood centrifugation $\left(6000 \mathrm{rpm}\right.$ for 15 minutes at $\left.25^{\circ} \mathrm{C}\right)$. Briefly, Maxisorp plates (Nunc, Life Technologies, USA) were coated with $5 \mu \mathrm{g} / \mathrm{mL}$ of bovine PG (Sigma Aldrich, St. Louis, MO, USA) and nonspecific protein binding was blocked with $0.1 \%$ bovine serum albumin in phosphate buffered saline. Subsequently, plates were incubated with serum samples diluted 1:1000. Biotinylated anti-mouse IgG1 and IgG2a antibodies (BD Biosciences, San Jose, CA, USA) were used to detect heterologous anti-PG antibodies. Plates were then incubated with streptavidin (RD Systems, Minneapolis, MN, USA) and revealed by adding $\mathrm{H}_{2} \mathrm{O}_{2}$ and orthophenylenediamine (Sigma Aldrich, St. Louis, MO, USA).

2.5. Statistical Analysis. Results were presented as mean \pm standard deviation for parametric variables and the comparison among the groups was performed by $t$-test. For nonparametric variables, the results were presented as median and the comparison between the groups was performed by MannWhitney's test. Paired $t$-test was performed for antibody production. All data were analyzed using SigmaPlot software version 12.0 (Jandel Corporation, USA) and $P<0.05$ was considered significant.

\section{Results}

3.1. Arthritis Incidence and Clinical Score. As expected, animals from control group did not develop experimental arthritis. However, all animals immunized with three doses of bovine PG+DDA adjuvant developed the disease (Figure 1(a)). Arthritis onset was observed at day 51 and total clinical score increased in the arthritic group until day 70 (Figure 1(b)). Moreover, the median of the maximum score in the arthritic group was statistically significant in comparison to the healthy control group (Figure 1(c)).

3.2. Histopathological Analysis. Figure 2 shows the differences among the clinical scores observed in mice hind paws and forepaws during arthritis development. HE stained paw sections revealed important histological changes in the arthritic joints compared to the healthy ones. According to the scoring system, all animals from control group presented score 0 and there was no signal of inflammation in these animals (Figures $2(\mathrm{a})$ and $2\left(\mathrm{a}^{\prime}\right)$ ). The joint structure was preserved and characterized by a well-defined synovial space, cartilage presence, thin synovial membrane, and compact bone (Figure $2\left(\mathrm{a}^{\prime \prime}\right)$ ). Mice from arthritic group presented a variety of scores, ranging from 1 to 4 in each paw. Score 1 was characterized by only one inflamed joint (head arrows; Figures $2(b)$ and $2\left(b^{\prime}\right)$ ). No differences were observed in histological sections from paws with score 1; that is, all animals presented well preserved joint structures (Figure $\left.2\left(b^{\prime \prime}\right)\right)$. Score 2 was characterized by the presence of two or more affected joints in the paw (Figures 2(c) and $\left.2\left(c^{\prime}\right)\right)$. In this score, there was an inflammatory cell infiltrate and a slight thickening of the synovial membrane. However, it was still possible to observe the presence of the synovial space and well-preserved cartilage and bone tissue (Figure $2\left(c^{\prime \prime}\right)$ ). Score 3 was characterized by the inflammation of multiple joints including the ankle (lines; Figures 2(d) and $\left.2\left(\mathrm{~d}^{\prime}\right)\right)$. In this score, there was an inflammatory cells infiltrate that characterizes the initial pannus formation, which is the inflammatory tissue that invades the synovial space and promotes cartilage destruction and bone erosion (Figure $2\left(\mathrm{~d}^{\prime \prime}\right)$ ). However, bone tissue was still preserved in this score. Score 4 was characterized by accentuated erythema and edema throughout the foot and ankle, involving all joints, with consequent movement impairment (Figures 2(e) and $\left.2\left(\mathrm{e}^{\prime}\right)\right)$. Inflammation and joint destruction were evident and were characterized by pannus formation, synovial membrane thickening, cartilage destruction, and bone erosion in paws with score 4 (Figure $2\left(\mathrm{e}^{\prime \prime}\right)$ ).

3.3. Production of Cytokines. Compared to the control group, spleen cells from arthritic mice produced significantly higher levels of IL-2 and the proinflammatory cytokines TNF$\alpha$, IL-6, IFN- $\gamma$, and IL-17 when restimulated in vitro with the specific antigen (Figures 3, 4, and 5). Interestingly, arthritic animals also produced significant levels of IL-5 and IL-10 anti-inflammatory cytokines in response to in vitro stimulation with PG (Figure 6). Results from nonstimulated cultures showed that there was spontaneous production of all cytokines in the arthritic animals, but not in the healthy ones (Figures 3, 4, 5, and 6). However, polyclonal stimulation of spleen cells with ConA triggered significant increase only in IL-6, IL-17, and IL-10 production by spleen cells from the arthritic group compared to the control one.

3.4. Production of Anti-PG Antibodies. The experimental arthritis induced by bovine PG determined production of both IgG1 and IgG2a anti-PG antibodies, with higher production of IgG1 (Figure 7). The levels of these specific antibodies increased significantly and progressively after the first immunization with PG+DDA (day 1). After reaching the maximum level around day 41 , antibody production was maintained until euthanasia at day 70. As expected, control animals that were not immunized with PG+DDA did not produce specific antibodies against bovine PG (data not shown).

\section{Discussion}

There are several experimental models of rheumatoid arthritis that contribute to understand the mechanisms involved in this disease [24]. Experimental arthritis models induced 


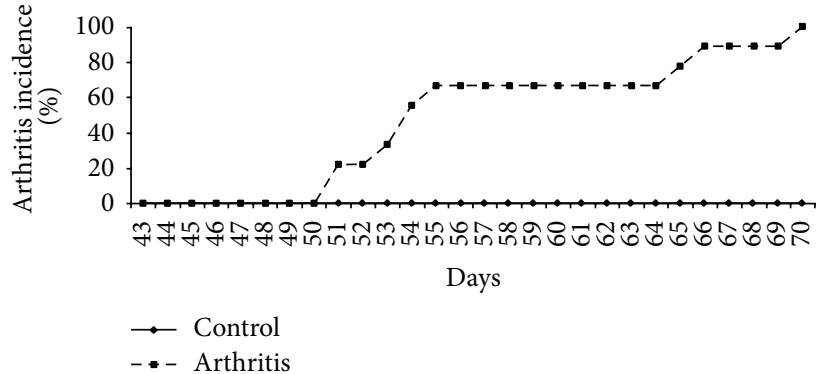

(a)

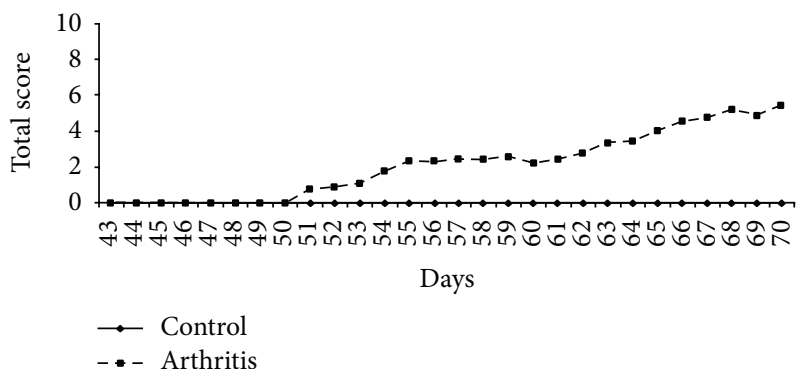

(b)

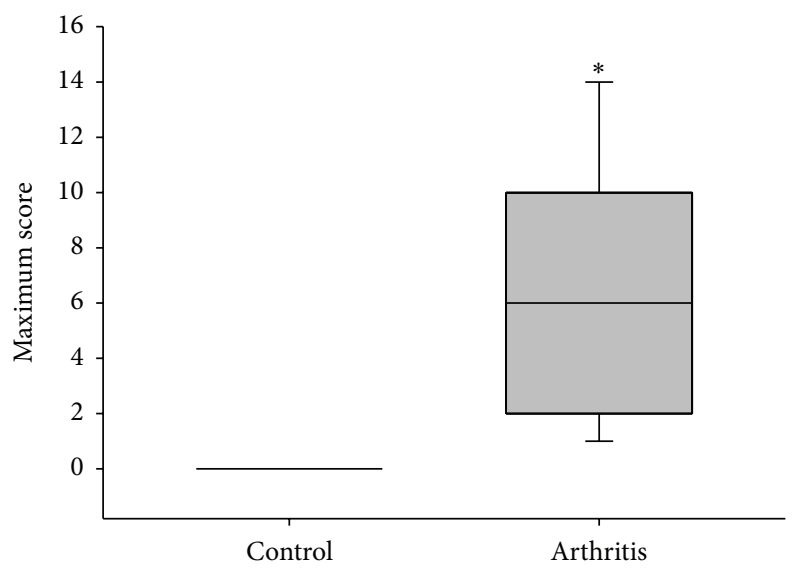

(c)

Figure 1: Arthritis incidence (a) total clinical score (b) and maximum clinical score (c) in mice with bovine proteoglycan-induced arthritis. Female BALB/c retired breeder mice were immunized with three doses of bovine PG associated with DDA adjuvant, 21-day interval. Clinical score was daily evaluated after the third immunization. ${ }^{*} P<0.05$ compared to control.

by cartilage components have been extensively studied, primarily the induction of arthritis by collagen and proteoglycan (PGIA). Considering the clinical and histopathological characteristics of the disease, this model shares many similarities with human arthritis. The development of arthritis in PGIA is attributed to a cross-reaction against foreign PG and mice self-PG [25]. In this experimental model, the disease is usually triggered by injections of human PG associated with a strong adjuvant. The PG can be extracted from the cartilage of various origins, but human PG is considered the most arthritogenic one. In this context and considering that PG purification is a complex and laborious process, we determined the arthritogenicity of a commercial source of bovine PG. This evaluation was done by immunization of $\mathrm{BALB} / \mathrm{c}$ retired breeders with three doses of bovine $\mathrm{PG}$ emulsified with DDA. In spite of the advanced age of these animals, no spontaneous arthritis was observed. According to Besenyei et al. [26], approximately 0.5 to $1.0 \%$ of retired breeder $\mathrm{BALB} / \mathrm{c}$ mice can develop the disease spontaneously.

Immunization with the commercial bovine PG was very effective to induce arthritis. A 100\% incidence was observed in the majority of the experiments as has been described with human PG $[13,23]$. In terms of clinical disease, we observed slightly lower scores than the ones described for human PG. However, this finding was equally described by other authors that employed bovine PG [23, 27]. In spite of this, the histopathological analysis revealed the presence of very typical arthritic histological alterations as inflammatory infiltrates, synovial membrane thickening, pannus formation, cartilage destruction, and bone erosion. These features are very similar to the ones described by other authors in PGIA and also in human arthritis $[13,28]$. This parallelism indicates that bovine PG can be further explored as another source of antigen to study arthritis. The efficacy of this bovine PG to induce murine arthritis is probably related, among other things, to the adopted adjuvant. As nicely described by Hanyecz et al. [23], the arthritogenicity of bovine PG was significantly incremented when it was combined with DDA. We also believe that the employment of $\mathrm{BALB} / \mathrm{c}$ retired breeders contributed a lot to this achievement. According to Tarjanyi et al. [29], these old animals are very prone to arthritis development.

Immunization with this commercial product also induced significant production of IgG1 and IgG2b antibovine PG antibodies. Even though we were not able to assess a possible cross-reactivity of these antibodies with murine PG, we believe that it exists and is underlying the arthritogenicity of bovine PG to mice. This assumption is mainly based on structural and comparative biochemical studies and on arthritogenicity for mice. In this context, 


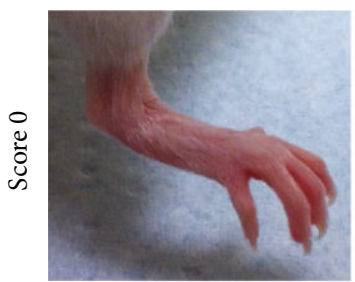

(a)

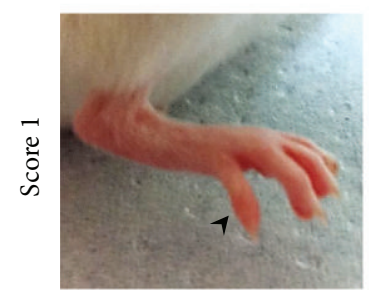

(b)

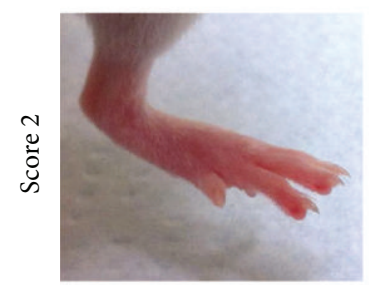

(c)

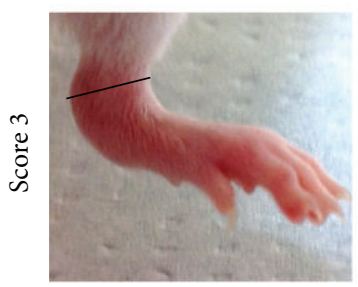

(d)

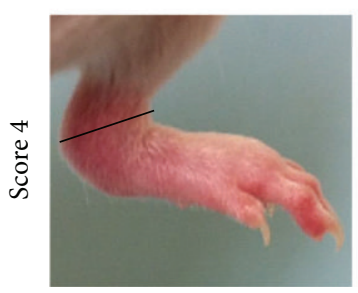

(e)

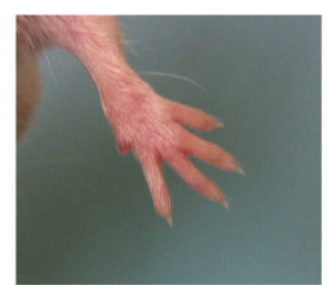

$\left(a^{\prime}\right)$

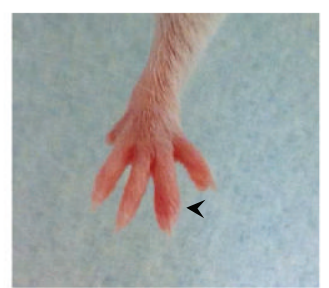

$\left(b^{\prime}\right)$

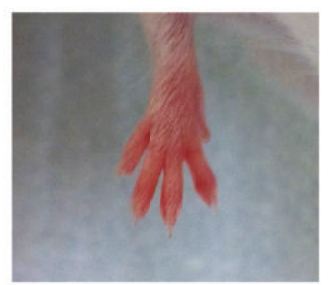

$\left(c^{\prime}\right)$

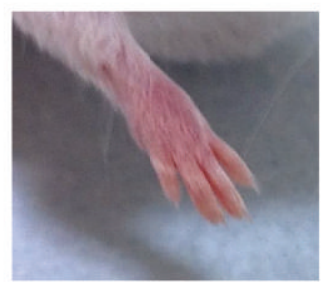

$\left(d^{\prime}\right)$

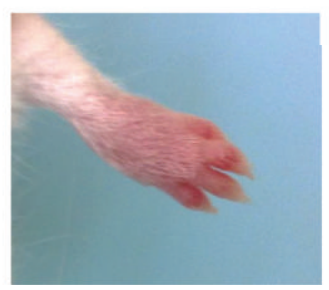

$\left(\mathrm{e}^{\prime}\right)$
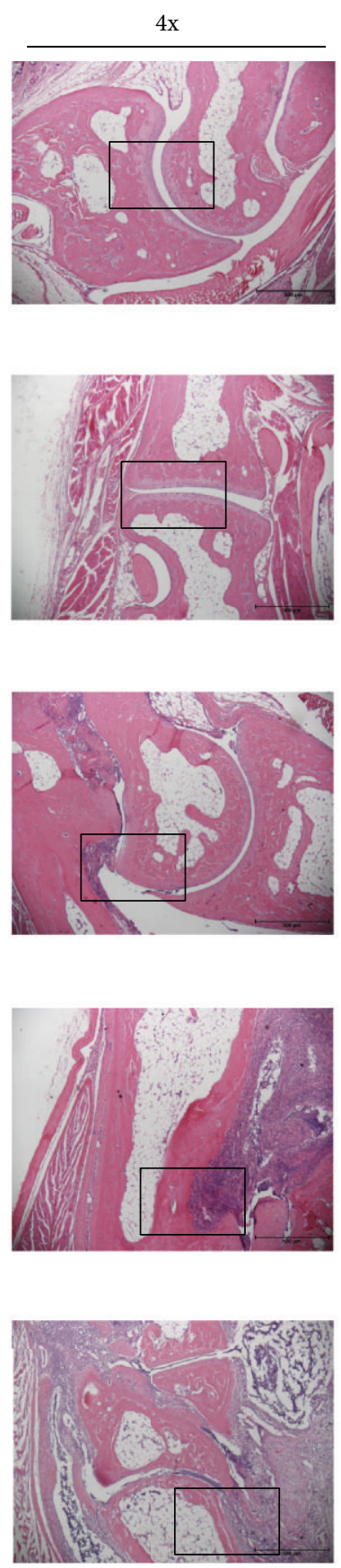

FIGURE 2: Representative clinical scores of hind paws (first column) and forepaws (second column) and histological sections of mice joints (third and fourth columns) with arthritis induced by bovine PG. Female BALB/c retired breeder mice paws were collected 70 days after the disease induction. The rectangles represent the regions highlighted in the fourth column. Head arrows indicate single joint inflammation; lines indicate ankle thickening; filled arrows indicate the synovial membrane. SS: synovial space; C: cartilage; B: bone; I: inflammatory infiltrate; P: pannus; BE: bone erosion.

Walcz et al. [30] demonstrated that murine and bovine PG core protein share $72.5 \%$ homology. The arthritogenic potential of distinct PG sources was checked in mice. Interestingly, arthritogenicity or its absence was associated with the ability to induce or not, respectively, the production of cross-reactive antibodies [31].
An aspect that deserves further elucidation is the degree of glycosylation present in this commercial PG. It has been strongly emphasized that PG deglycosylation is fundamental to achieve arthritogenicity [13, 32]. However, we believe that this preparation is not devoid of polysaccharides. This hypothesis is based on references specified by the company 

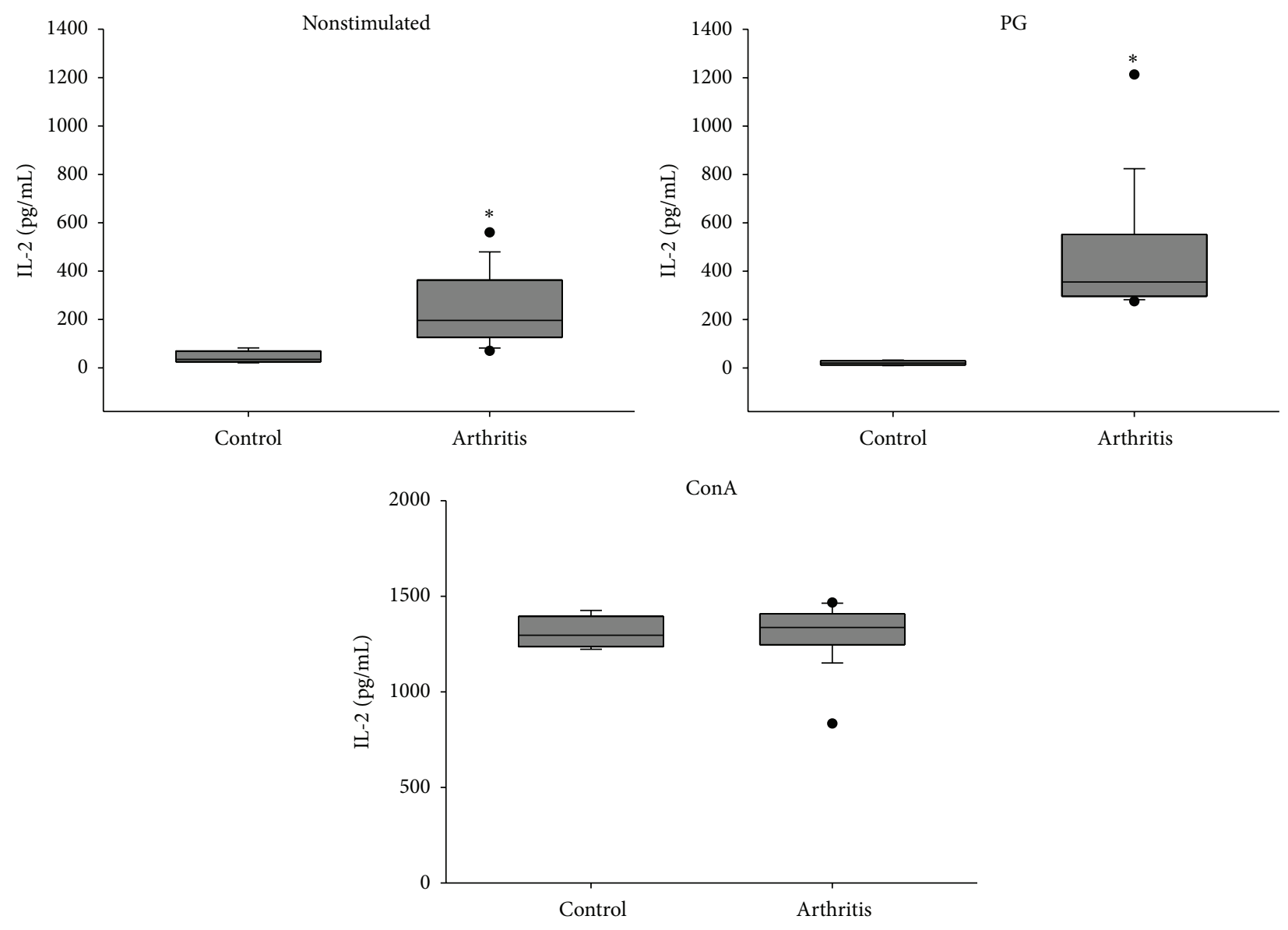

Figure 3: IL-2 production by spleen cells from BALB/c retired breeder mice with bovine proteoglycan-induced arthritis. Spleen cells were in vitro stimulated with PG and ConA and incubated for 48 hours. ${ }^{*} \mathrm{P}<0.05$ compared to the respective control.

that commercializes the product and also in information described by authors that utilized this product. According to Tham et al. [33], this commercial product contains $86 \%$ of chondroitin sulfate, $8 \%$ protein, $6 \%$ keratan sulfate, and less than $1 \%$ hyaluronic acid and it was able to enhance the survival of neural stem cells. In the central nervous system, chondroitin sulfate proteoglycan (CSPG) is the most prevalent PG and CS removal with chondroitinase reduces neural stem cells proliferation and neurogenesis. Also, according to Antonopoulos et al. [34], PG isolated by urea procedure is probably found in PG subunits instead of PG complexes form due to its gel chromatographic pattern. PG subunits could expose the G1 domain and the link protein, which are highly arthritogenic [27, 35]. Antonopoulos et al. [34] also demonstrated that urea did not cause PG degradation. It is possible to think that this organic compound could interfere in PG structure and protein solubility exposing some core protein epitopes and, therefore, become able to induce experimental arthritis.

Results from cytokine production by spleen cells in vitro stimulated with PG showed that arthritic animals, but not healthy ones, produced high levels of IL-2, TNF- $\alpha$, IL-6, IFN$\gamma$, and IL-17. Spleen cells from arthritic mice were already producing higher levels of IL-2 than the healthy ones. Also, addition of PG to the cultures determined a significant increase in the production of this cytokine in the arthritic group. As a very good correlation has been established between IL-2 level and T-cell proliferation index, in either up- [36] or downregulation [37], our results indicate the occurrence of a specific proliferative process in the spleen. The higher production of TNF- $\alpha$, IL- 6 , IFN- $\gamma$, and IL-17 was expected and corroborates with their arthritogenic potential observed in humans [38] and in animal models [24]. The production of IL- 6 and TNF$\alpha$ is related to the immunopathogenesis and maintenance of RA. These cytokines are also responsible for hyperalgesia caused by mechanical stimulus in this disease. According to Schaible et al. [38], these proinflammatory cytokines act on nerve cells responsible for the nociceptive stimuli during joint movement. These authors showed that drugs which neutralize the action of TNF- $\alpha$ promoted reduction of pain and inflammation in rats with adjuvant-induced arthritis. A similar result was found concerning IL-6 neutralization. 

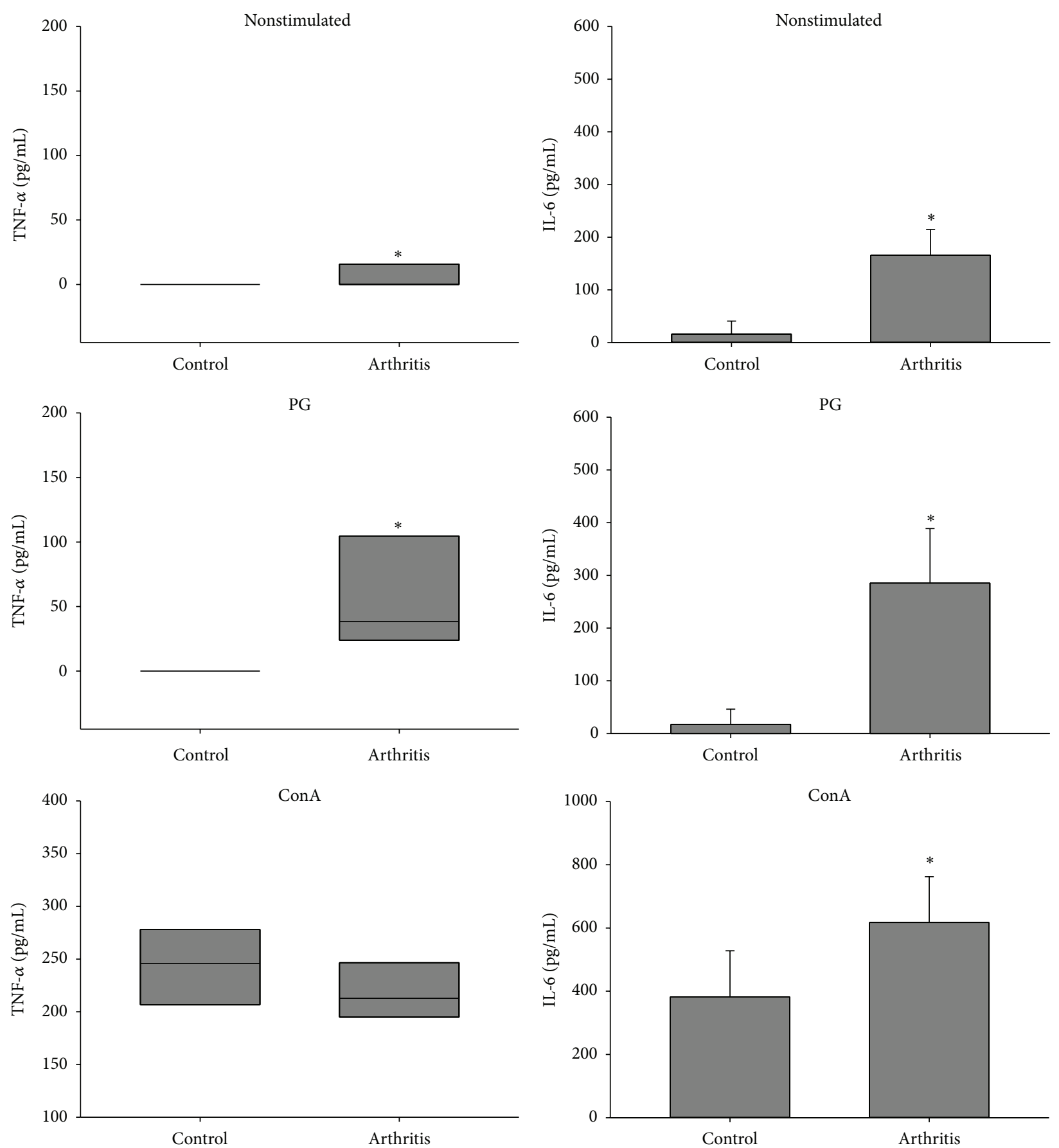

FIGURE 4: TNF- $\alpha$ and IL- 6 production by spleen cells from BALB/c retired breeder mice with bovine proteoglycan-induced arthritis. Spleen cells were in vitro stimulated with PG and ConA and incubated for 48 hours. ${ }^{*} P<0.05$ compared to the respective control.

Thus, drugs whose action mechanisms are based on TNF$\alpha$, IL-6, and IL-1 neutralization have been extensively studied and some of them such as TNF- $\alpha$ and IL- 6 are already used for the treatment of RA clinical symptoms [39].

Many studies have considered the balance between the production of IL-17 and IFN- $\gamma$ as the key to understand the main immunopathogenic mechanisms involved in arthritis development. Considered a major proinflammatory cytokine in human arthritis and in most experimental models, IL-17 plays an important role in the establishment, maintenance, and progression of this disease [40-42]. Regarding this, studies have shown that the absence of IL-17 decreased the clinical symptoms of arthritis in different experimental models $[43,44]$. The role of IL-17 in PGIA is not clearly evaluated yet. However, it has been suggested that in this case the IFN- $\gamma$ is more important than IL-17 in the establishment 

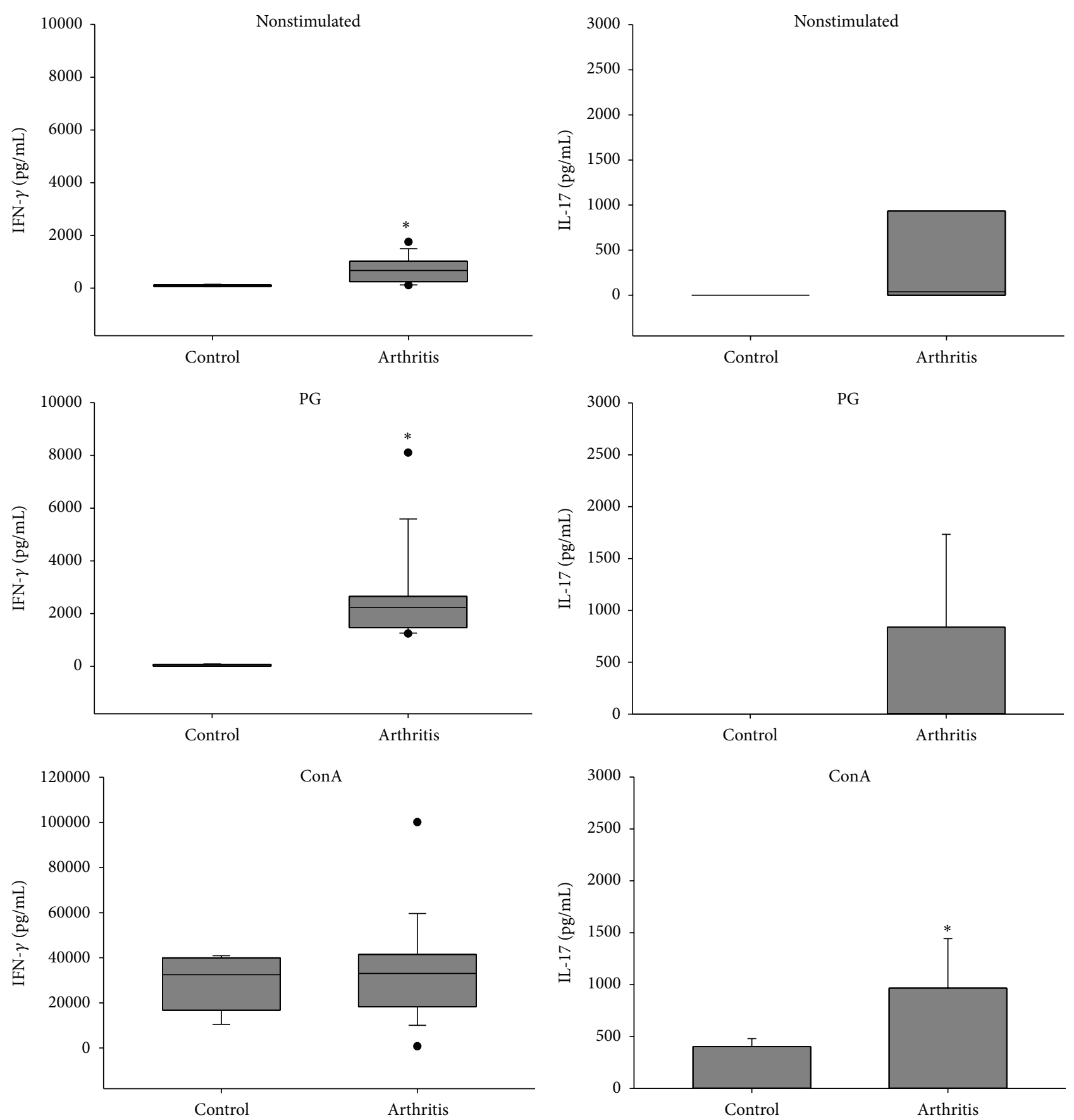

FIGURE 5: IFN- $\gamma$ and IL-17 production by spleen cells from BALB/c retired breeder mice with bovine proteoglycan-induced arthritis. Spleen cells were in vitro stimulated with PG and ConA and incubated for 48 hours. ${ }^{*} P<0.05$ compared to the respective control.

of the disease. Doodes et al. [21], using knockout mice, showed that IFN- $\gamma$ is essential for PGIA triggering in an IL-17 independent manner. The IFN- $\gamma$ represents a paradox in autoimmune arthritis. Although its pathogenic effect is well described, recent studies showed a protective effect of this cytokine in arthritis. Alzabin and Williams [45] carefully reviewed the role of effector $\mathrm{T}$ cells in autoimmune arthritis. By analyzing the results of several experimental models, the authors demonstrated the protective role of IFN- $\gamma$.
The administration of this cytokine that is, theoretically, proinflammatory, decreased clinical signals in different arthritis models. For example, genetically modified animals which were not able to produce IFN- $\gamma$ presented an exacerbated collagen-induced arthritis [46]. However, it has been also reported that animals that did not produce this cytokine were less susceptible to PGIA [47].

The specific in vitro stimulation of spleen cells also triggered production of anti-inflammatory cytokines such 

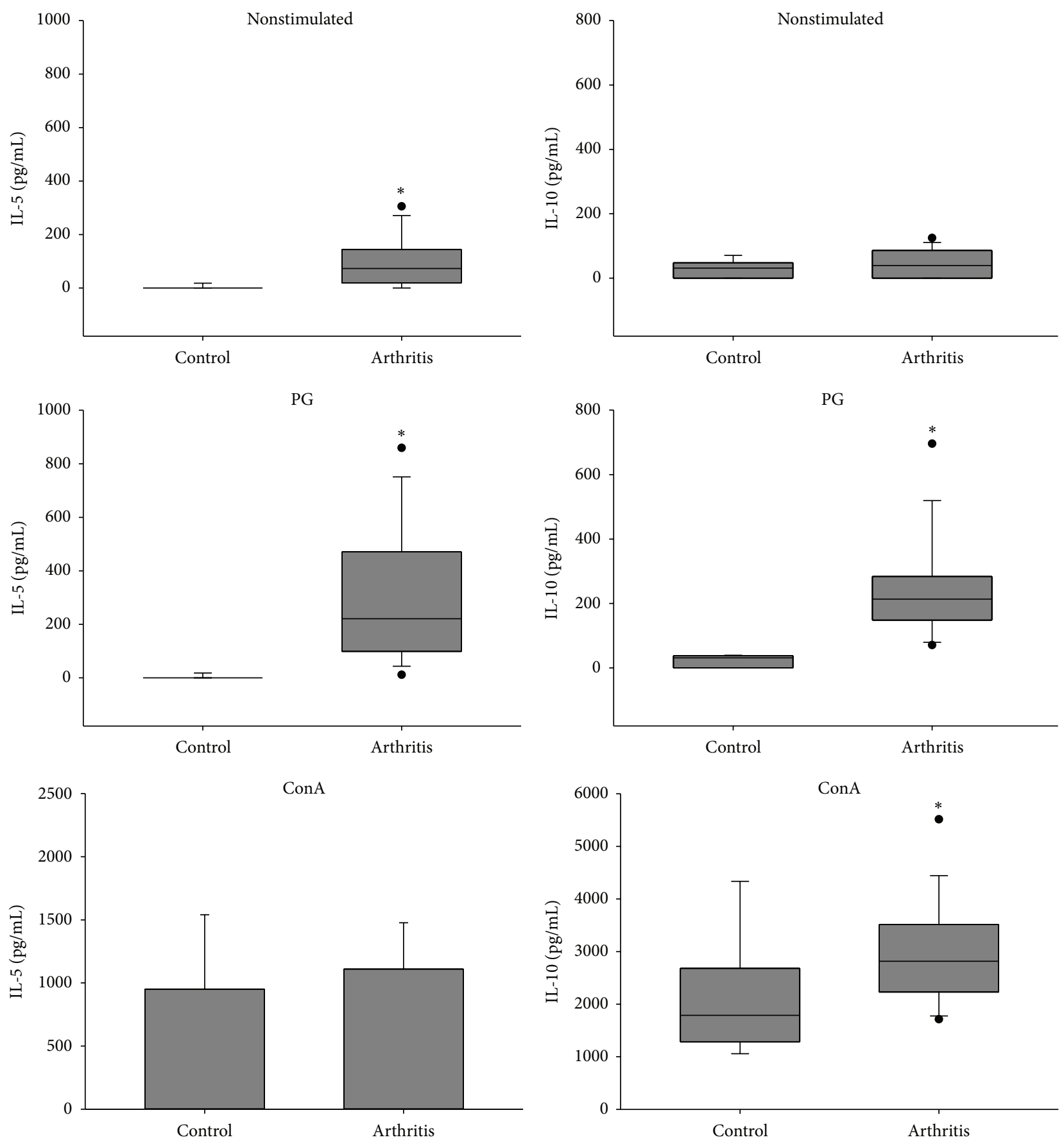

FIGURE 6: IL-5 and IL-10 production by spleen cells from BALB/c retired breeder mice with bovine proteoglycan-induced arthritis. Spleen cells were in vitro stimulated with PG and ConA and incubated for 48 hours. ${ }^{*} P<0.05$ compared to the respective control.

as IL-5 and IL-10. Although RA is considered a disease characterized by predominant Th1 pattern, studies indicate that Th2 cytokines such as IL- 4 and IL-10 also contribute to the immunopathogenesis of the disease and may also be related to the stage of disease development [48]. According to Gerli et al. [49], there is a high production of IL-4 and IL-10 by $\mathrm{T}$ cells from peripheral blood of patients in earlier stages of arthritis, but this production decreases significantly in later stages, contributing to disease progression and joint destruction in the chronic phase. Our results are, therefore, similar to the mixed Th1/Th2 pattern already shown in humans and in PGIA model [31]. An interesting aspect was observed in nonstimulated spleen cell cultures from arthritic animals when compared to control group. The arthritic group produced detectable levels of IFN- $\gamma$, TNF- $\alpha$, IL-6, IL-17, IL-5, and IL-10 even in the absence of the specific 


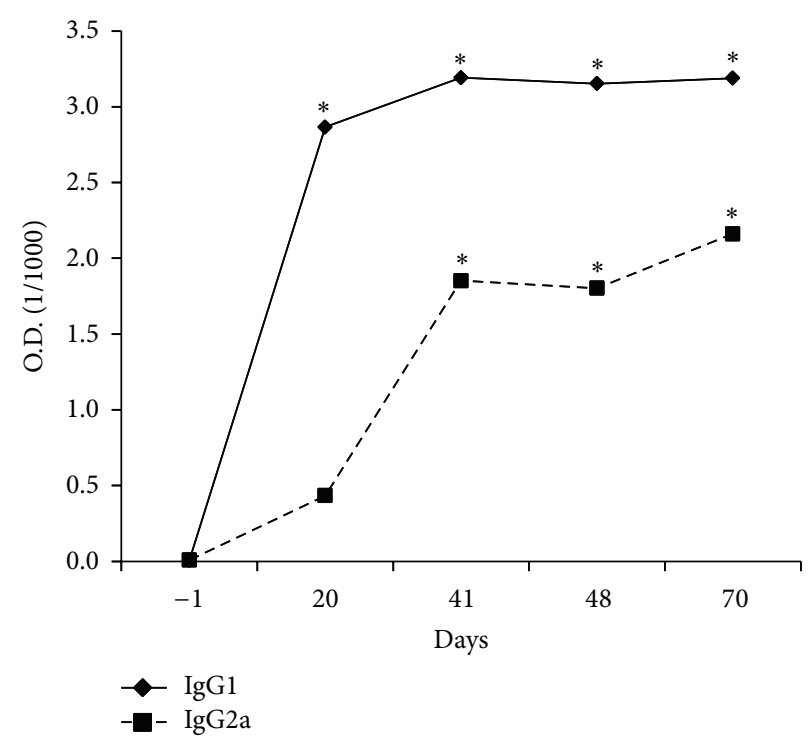

Figure 7: IgG1 and IgG2a serum levels from BALB/c retired breeder mice with bovine proteoglycan-induced arthritis. Blood samples were obtained two days before each PG+DDA immunization (days -1, 20, and 41), seven days after the third immunization (day 48), and after euthanasia (day 70$){ }^{*} P<0.05$ compared to day -1 of the same group.

stimulus. This spontaneous production, which might be more properly called endogenous production, could result from the intense immune response activation and cytokine secretion by effector cells that are significantly occurring.

Some interesting results mainly related to IL-17 were also detected in spleen cell cultures stimulated with ConA. In this case, we highlight the fact that the production of this cytokine after polyclonal stimulation was very similar to that induced by specific antigen stimulation. This finding is different from the ones usually obtained after polyclonal activation. The stimulation with mitogens is usually associated with induction of significantly higher cytokine production than the specific stimulus. However, recently, Doodes et al. [21] observed that the production of IL-17 and IFN- $\gamma$ in response to specific stimulus is extremely high, reaching levels greater than $2000 \mathrm{pg} / \mathrm{mL}$ in the PGIA model. Similarly high levels of IL-17 were observed in human studies. Leipe et al. [50] evaluated the importance of IL-17 in autoimmune arthritis and found that purified $\mathrm{T}$ cells from the peripheral blood of patients, in the early stage of the disease, produced very high levels of this cytokine. Furthermore, analysis of the production of IL-17 by mononuclear cells from peripheral blood of healthy individuals, in response to different mitogens, revealed that the level of this cytokine in response to ConA did not exceed $500 \mathrm{pg} / \mathrm{mL}$ [51].

\section{Conclusions}

Our results indicate that this commercial bovine $P G$ is highly arthritogenic for $\mathrm{BALB} / \mathrm{c}$ retired breeder mice. In addition, the disease induced by this reagent presents clinical symptoms and histopathological features that are very similar to those found in other arthritis models and also the human corresponding pathology. Taken together, these results suggest that this bovine PG can be used as an alternative source in PGIA for the study of many aspects of RA, including the immunopathogenesis of the disease and also the development of new therapies.

\section{Conflict of Interests}

The authors declare that there is no conflict of interests regarding the publication of this paper.

\section{Acknowledgments}

The authors are grateful to Brazilian financial support: Grant number 2011/17325-4, São Paulo State Foundation (FAPESP). Special thanks are due to Professor Gustavo Pompermaier Garlet, Daniele Santi Ceolin, and Patrícia de Sá Mortagua Germino from the Department of Biological Sciences, School of Dentistry of Bauru, São Paulo University (USP), Brazil, for the contribution to the histopathological analysis.

\section{References}

[1] WHO Scientific Group, "The burden of musculoskeletal conditions at the start of the new millennium," Technical Repors 919, World Health Organization, Geneva, Switzerland, 2003.

[2] J. B. Imboden, "The immunopathogenesis of rheumatoid arthritis," Annual Review of Pathology: Mechanisms of Disease, vol. 4, pp. 417-434, 2009.

[3] Y. W. Song and E. H. Kang, "Autoantibodies in rheumatoid arthritis: rheumatoid factors and anticitrullinated protein antibodies," QJM, vol. 103, no. 3, Article ID hcp165, pp. 139-146, 2009. 
[4] A. M. Gizinski and D. A. Fox, "T cell subsets and their role in the pathogenesis of rheumatic disease," Current Opinion in Rheumatology, vol. 26, no. 2, pp. 204-210, 2014.

[5] Z. Szekanecz and A. E. Koch, "Macrophages and their products in rheumatoid arthritis," Current Opinion in Rheumatology, vol. 19, no. 3, pp. 289-295, 2007.

[6] E. Choy, "Understanding the dynamics: pathways involved in the pathogenesis of rheumatoid arthritis," Rheumatology, vol. 51, no. 5, Article ID kes113, pp. 3-11, 2012.

[7] M. I. Koenders and W. B. Van Den Berg, "Translational minireview series on Th17 cells: are Thelper 17 cells really pathogenic in autoimmunity?" Clinical and Experimental Immunology, vol. 159, no. 2, pp. 131-136, 2010.

[8] B. Nakken, L. A. Munthe, Y. T. Konttinen et al., "B-cells and their targeting in rheumatoid arthritis-current concepts and future perspectives," Autoimmunity Reviews, vol. 11, no. 1, pp. 28-34, 2011.

[9] S. Oh, A. L. Rankin, and A. J. Caton, "CD4+CD25+ regulatory T cells in autoimmune arthritis," Immunological Reviews, vol. 233, no. 1, pp. 97-111, 2010.

[10] P. H. Wooley, J. R. Seibold, J. D. Whalen, and J. M. Chapdelaine, "Pristane-induced arthritis. The immunologic and genetic features of an experimental murine model of autoimmune disease," Arthritis and Rheumatism, vol. 32, no. 8, pp. 1022-1030, 1989.

[11] C. Vingsbo-Lundberg, N. Nordquist, P. Olofsson et al., "Genetic control of arthritis onset, severity and chronicity in a model for rheumatoid arthritis in rats," Nature Genetics, vol. 20, no. 4, pp. 401-404, 1998.

[12] M. Brenner, T. Laragione, and P. S. Gulko, "Arthritis severity locus Cia 4 is an early regulator of IL- 6 , IL- $1 \beta$, and NF- $\kappa$ B activators' expression in pristane-induced arthritis," Physiological Genomics, vol. 45, no. 13, pp. 552-564, 2013.

[13] T. T. Glant, A. Finnegan, and K. Mikecz, "Proteoglycaninduced arthritis: immune regulation, cellular mechanisms, and genetics," Critical Reviews in Immunology, vol. 23, no. 3, pp. 199250, 2003.

[14] D. D. Brand, A. H. Kang, and E. F. Rosloniec, "Immunopathogenesis of collagen arthritis," Springer Seminars in Immunopathology, vol. 25, no. 1, pp. 3-18, 2003.

[15] J. Keffer, L. Probert, H. Cazlaris et al., "Transgenic mice expressing human tumour necrosis factor: a predictive genetic model of arthritis," EMBO Journal, vol. 10, no. 13, pp. 4025-4031, 1991.

[16] X. Zhu, L. Xiao, R. Huo et al., "Cyr61 is involved in neutrophil infiltration in joints by inducing IL-8 production by fibroblastlike synoviocytes in rheumatoid arthritis," Arthritis Research and Therapy, vol. 15, no. 6, p. R187, 2013.

[17] C. H. Sarithakumari and G. M. Kurup, "Alginic acid isolated from Sargassumwightii exhibits anti-inflammatory potential on type II collagen induced arthritis in experimental animals," International Immunopharmacology, vol. 17, no. 4, pp. 1108-1115, 2013.

[18] J. Woo, M. P. Vierboom, H. Kwon et al., "PDL241, a novel humanized monoclonal antibody, reveals CD319 as a therapeutic target for rheumatoid arthritis," Arthritis Research \& Therapy, vol. 15, no. 6, p. R207, 2013.

[19] T. Eneljung, S. Tengvall, P. Jirholt et al., "Antigen-specific gene therapy after immunisation reduces the severity of collageninduced arthritis," Clinical and Developmental Immunology, vol. 2013, Article ID 345092, 11 pages, 2013.
[20] E. I. Buzás, A. Végvári, Y. M. Murad, A. Finnegan, K. Mikecz, and T. T. Glant, "T-cell recognition of differentially tolerated epitopes of cartilage proteoglycan aggrecan in arthritis," Cellular Immunology, vol. 235, no. 2, pp. 98-108, 2005.

[21] P. D. Doodes, Y. Cao, K. M. Hamel et al., "IFN- $\gamma$ regulates the requirement for IL-17 in proteoglycan-induced arthritis," Journal of Immunology, vol. 184, no. 3, pp. 1552-1559, 2010.

[22] A. Finnegan, M. J. Grusby, C. D. Kaplan et al., "IL-4 and IL-12 regulate proteoglycan-induced arthritis through stat-dependent mechanisms," Journal of Immunology, vol. 169, no. 6, pp. 3345-3352, 2002.

[23] A. Hanyecz, S. E. Berlo, S. Szántó, C. P. M. Broeren, K. Mikecz, and T. T. Glant, "Achievement of a synergistic adjuvant effect on arthritis induction by activation of innate immunity and forcing the immune response toward the Th1 phenotype," Arthritis and Rheumatism, vol. 50, no. 5, pp. 1665-1676, 2004.

[24] W. B. van den Berg, "Lessons from animal models of arthritis over the past decade," Arthritis Research and Therapy, vol. 11, no. 5 , p. 250, 2009.

[25] T. T. Glant and K. Mikecz, "Proteoglycan aggrecan-induced arthritis: a murine autoimmune model of rheumatoid arthritis," Methods in Molecular Medicine, vol. 102, pp. 313-338, 2004.

[26] T. Besenyei, A. Kadar, B. Tryniszewska et al., "Non-MHC risk alleles in rheumatoid arthritis and in the syntenic chromosome regions of corresponding animal models," Clinical and Developmental Immunology, vol. 2012, Article ID 284751, 14 pages, 2012.

[27] J. Y. Leroux, A. Guerassimov, A. Cartman et al., "Immunity to the G1 globular domain of the cartilage proteoglycan aggrecan can induce inflammatory erosive polyarthritis and spondylitis in BALB/c mice but immunity to G1 is inhibited by covalently bound keratan sulfate in vitro and in vivo," The Journal of Clinical Investigation, vol. 97, no. 3, pp. 621-632, 1996.

[28] R. C. Jeffery, "Clinical features of rheumatoid arthritis," Medicine, vol. 38, no. 4, pp. 167-171, 2010.

[29] O. Tarjanyi, F. Boldizsar, P. Nemeth, K. Mikecz, and T. T. Glant, "Age-related changes in arthritis susceptibility and severity in a murine model of rheumatoid arthritis," Immunity and Ageing, vol. 11, no. 6, p. 8, 2009.

[30] E. Walcz, F. Deak, P. Erhardt et al., "Complete coding sequence, deduced primary structure, chromosomal localization, and structural analysis of murine aggrecan," Genomics, vol. 22, no. 2, pp. 364-371, 1994.

[31] K. Holló, T. T. Glant, M. Garzó, A. Finnegan, K. Mikecz, and E. Buzás, "Complex pattern of Th1 and Th2 activation with a preferential increase of autoreactive Th1 cells in BALB/c mice with proteoglycan (aggrecan)-induced arthritis," Clinical and Experimental Immunology, vol. 120, no. 1, pp. 167-173, 2000.

[32] T. T. Glant, M. Radacs, G. Nagyeri et al., "Proteoglycan-induced arthritis and recombinant human proteoglycan aggrecan G1 domain-induced arthritis in $\mathrm{BALB} / \mathrm{c}$ mice resembling two subtypes of rheumatoid arthritis," Arthritis and Rheumatism, vol. 63, no. 5, pp. 1312-1321, 2011.

[33] M. Tham, S. Ramasamy, H. T. Gan et al., "CSPG is a secreted factor that stimulates neural stem cell survival possibly by enhanced EGFR signaling," PLoS ONE, vol. 5, no. 12, Article ID e15341, 2010.

[34] C. A. Antonopoulos, I. Axelsson, D. Heinegard, and S. Gardell, "Extraction and purification of proteoglycans from various types of connective tissue," Biochimica et Biophysica Acta, vol. 338, no. 1, pp. 108-119, 1974.

[35] Y.Zhang, A. Guerassimov, J. Leroux et al., "Induction of arthritis in $\mathrm{BALB} / \mathrm{c}$ mice by cartilage link protein: involvement of 
distinct regions recognized by $\mathrm{T}$ and $\mathrm{B}$ lymphocytes," American Journal of Pathology, vol. 153, no. 4, pp. 1283-1291, 1998.

[36] J. Chen, H. Wu, Q. Wang et al., "Ginsenoside metabolite compound $\mathrm{K}$ alleviates adjuvant-induced arthritis by suppressing $\mathrm{T}$ cell activation," Inflammation, 2014.

[37] A. Poosarla, R. DN, R. R. Athota, and V. G. Sunkara, "Modulation of $\mathrm{T}$ cell proliferation and cytokine response by Plumbagin, extracted from Plumbago zeylanica in collagen induced arthritis," BMC Complementary and Alternative Medicine, vol. 11, p. 114, 2011.

[38] H. G. Schaible, G. S. von Banchet, M. K. Boettger et al., "The role of proinflammatory cytokines in the generation and maintenance of joint pain: annals of the New York Academy of Sciences," Annals of the New York Academy of Sciences, vol. 1193, pp. 60-69, 2010.

[39] D. L. Scott, "Biologics-based therapy for the treatment of rheumatoid arthritis," Clinical Pharmacology and Therapeutics, vol. 91, no. 1, pp. 30-43, 2012.

[40] M. Chabaud, P. Garnero, J. Dayer, P. Guerne, F. Fossiez, and P. Miossec, "Contribution of interleukin 17 to synovium matrix destruction in rheumatoid arthritis," Cytokine, vol. 12, no. 7, pp. 1092-1099, 2000.

[41] E. Lubberts, P. Schwarzenberger, W. Huang et al., "Requirement of IL-17 receptor signaling in radiation-resistant cells in the joint for full progression of destructive synovitis," Journal of Immunology, vol. 175, no. 5, pp. 3360-3368, 2005.

[42] M. E. Truchetet, M. D. Mossalayi, and K. Boniface, "IL-17 in the rheumatologist's line of sight," Biomed Research International, vol. 2013, Article ID 295132, 18 pages, 2013.

[43] E. Lubberts, M. I. Koenders, B. Oppers-Walgreen et al., "Treatment with a neutralizing anti-murine interleukin-17 antibody after the onset of collagen-induced arthritis reduces joint inflammation, cartilage destruction, and bone erosion," Arthritis and Rheumatism, vol. 50, no. 2, pp. 650-659, 2004.

[44] K. A. Bush, K. M. Farmer, J. S. Walker, and B. W. Kirkham, "Reduction of joint inflammation and bone erosion in rat adjuvant arthritis by treatment with interleukin-17 receptor IgG1 Fc fusion protein," Arthritis and Rheumatism, vol. 46, no. 3, pp. 802-805, 2002.

[45] S. Alzabin and R. O. Williams, "Effector T cells in rheumatoid arthritis: lessons from animal models," FEBS Letters, vol. 585, no. 23, pp. 3649-3659, 2011.

[46] J. Lee, J. Lee, M. Park et al., "Interferon gamma suppresses collagen-induced arthritis by regulation of Th17 through the induction of indoleamine-2,3-deoxygenase," PLoS ONE, vol. 8, no. 4, Article ID e60900, 2013.

[47] C. Kaplan, J. C. Valdez, R. Chandrasekaran et al., "Th1 and Th2 cytokines regulate proteoglycan-specific autoantibody isotypes and arthritis," Arthritis Research, vol. 4, no. 1, pp. 54-58, 2002.

[48] J. A. van Roon, C. M. Verhoef, J. L. van Roy et al., "Decrease in peripheral type 1 over type $2 \mathrm{~T}$ cell cytokine production in patients with rheumatoid arthritis correlates with an increase in severity of disease," Annals of the Rheumatic Diseases, vol. 56, no. 11, pp. 656-660, 1997.

[49] R. Gerli, O. Bistoni, A. Russano et al., "In vivo activated T cells in rheumatoid synovitis. Analysis of Th1- and Th2-type cytokine production at clonal level in different stages of disease," Clinical and Experimental Immunology, vol. 129, no. 3, pp. 549-555, 2002.

[50] J. Leipe, M. Grunke, C. Dechant et al., "Role of Th17 cells in human autoimmune arthritis," Arthritis and Rheumatism, vol. 62 , no. 10 , pp. $2876-2885,2010$.
[51] A. Lenarczyk, J. Helsloot, K. Farmer, L. Peters, A. Sturgess, and B. Kirkham, "Antigen-induced IL-17 response in the peripheral blood mononuclear cells (PBMC) of healthy controls," Clinical and Experimental Immunology, vol. 122, no. 1, pp. 41-48, 2000. 


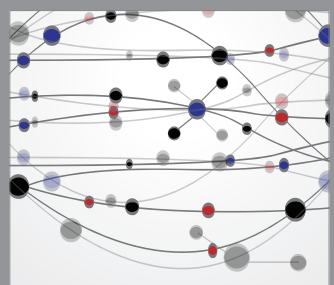

The Scientific World Journal
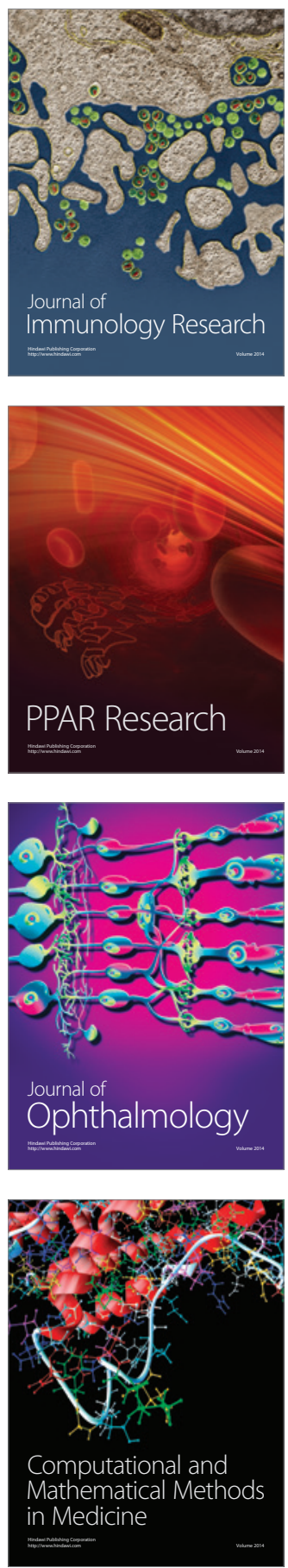

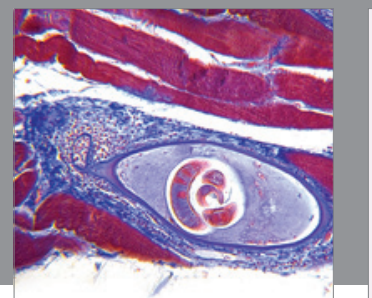

Gastroenterology

Research and Practice
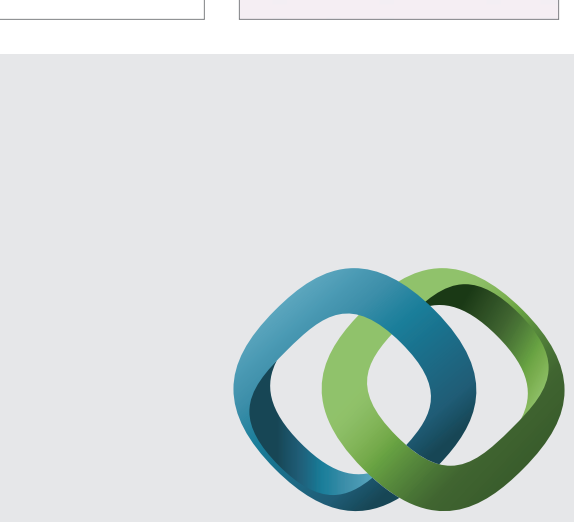

\section{Hindawi}

Submit your manuscripts at

http://www.hindawi.com
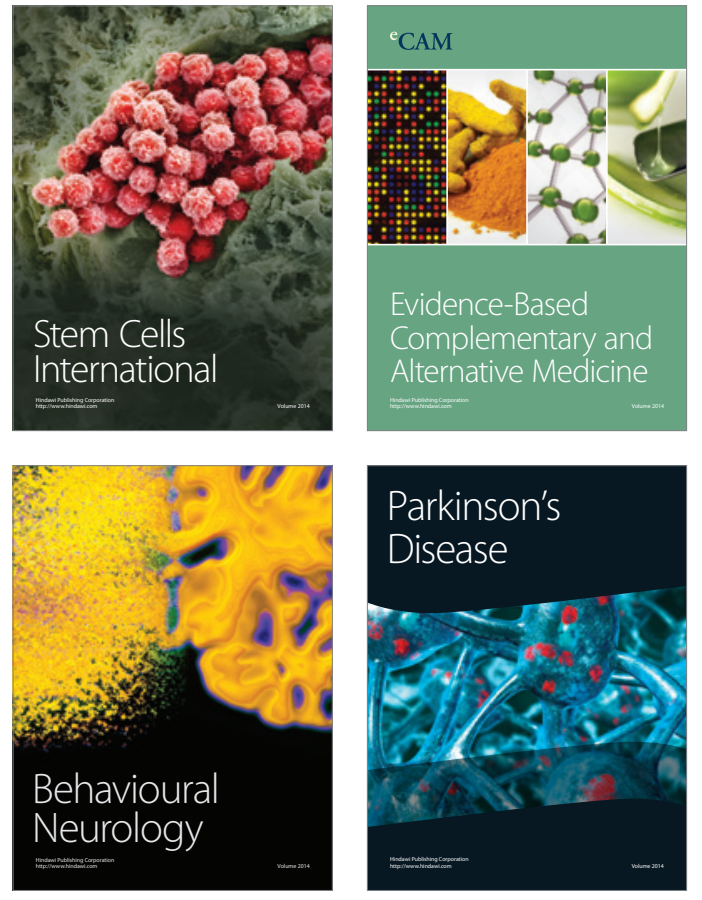
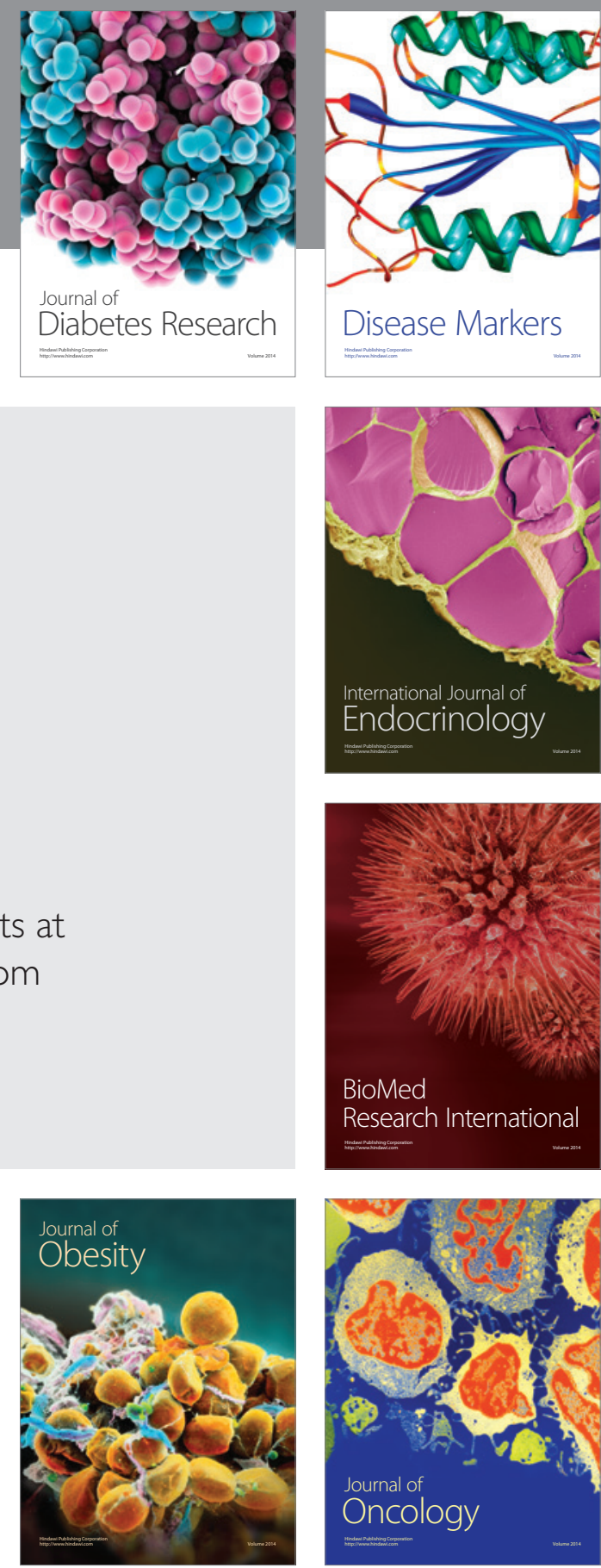

Disease Markers
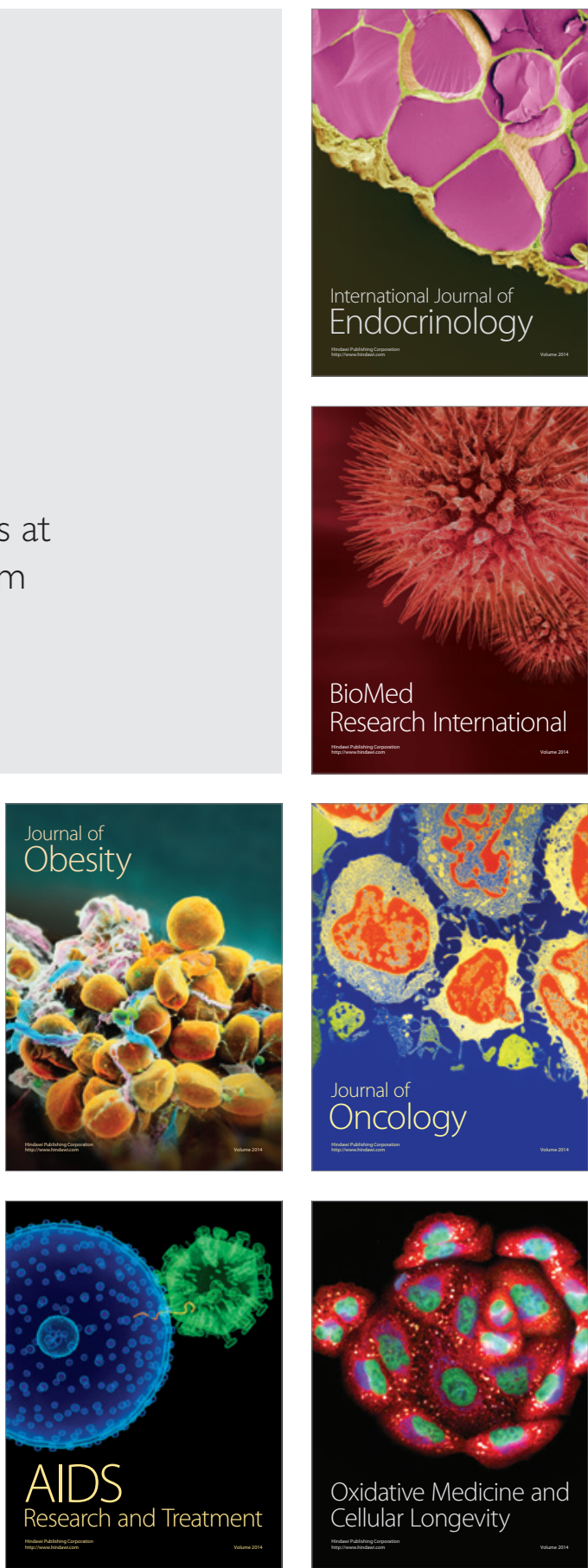